\title{
DEVELOPMENT OF ULTRÄSONIC EXAMINATION METHODS \\ FOR AUSTENITIC STAINLESS STEEL WELD INSPECTION
}

by

\section{MASTER}

S. J. Mech, T. E. Michaels, J. S. Emmons, and M. M. Sugiyama

August, 1977

\begin{abstract}
Signal acquisition and analysis techniques were developed to permit locating notches placed in an austenitic stainless steel pipe weld. UItrasonic techniques presented here utilized refracted longitudinal and shear inspection beons with a dual element, pitch-catch transducer. Sources of grain noise and the metallurgical implications of inspecting austenitic stainless steel welds are reviewed. Data were analyzed in both time and frequency donains, and a simple frequency moment algorithm was developed which is sensitive to defect signal and somewhat insensitive to grain noise. Data were also used to train an adaptive leaming network (ALN) and the results obtained demonstrate only slight sensitivity to grain noise.
\end{abstract}

This Notice

This report was prepared as an account of work Sponsored by the United States Government Neither the Ented States nor the United States Department of Energy, nor any of therr employees, nor any of their contractors, subcontractors, or their employees, makes any warranty, express or implied, or assumes any legal
lability or responsibility for the acouracy, or usefuiness of any information, appacy, completeness process disclosed, or represents that ts use product or infrunge prvately owned nghts

\section{Copyright Notice}

"By acceptance of this article, the Publisher and/or recipient acknowledges the U.S. Government's right to retain a nonexclusive, royalty-free license in and to any copyright covering this paper."

This paper is based on work performed by the Hanford Engineering Development Laboratory, Richland, Washington, operated by the Westinghouse Hanford Company, a subsidiary of Westinghouse Electric Corporation, under United States Energy Research and Development Administration Contract EY-76-C-142170. 


\section{DISCLAIMER}

Portions of this document may be illegible in electronic image products. Images are produced from the best available original document. 
DEVELOPMENT OF ULTRASONIC EXAMINATION METHODS

FOR AUSTENITIC. STAINLESS STEEL WELD INSPECTION

by

S. J. Mech, T. E. Michaels, J. S. Emmons, and M. M. Sugiyama

\section{INTRODUCTION}

Development of more effective methods for ultrasonic examination of welds in austenitic stainless steel components is an important objective in the nuclear industry. The specific objective of this program is to develop ultrasonic methods for pre-service and in-service inspection of austenitic stainless steel welds in certain Fast Flux Test Facility (FFTF) pipes.

The challenge is to develop techniques which find defects but ignore variations associated with the normal cast type microstructure of the weld zone. This study was directed at gathering data on a welded pipe section with notches used to simulate defects, and is another example of computer acquisition and analysis techniques of ultrasonic data. (1) Various analysis methods were compared to find signal analysis algorithms sensitive to circumferential location of the notches. For the overall problem, we classify algorithms in two broad categories. First, we need an inspection scheme to quickly scan a section to find all potential defect locations. In the second category, we need techniques to classify signals in order to size defects identified by the first method. This report discusses results for the first category. The program is continuing and the second category will be reported as significant results become available.

\section{AUSTENITIC STAINLESS STEEL MICROSTRUCTURE.}

For austenitic stainless steel welds, the microstructure dominates the wave propagation response. (2) The weld zone is characterized by large columnar grains (dendrites) which result from the slow cooling and directional 
solidification during welding. Wave velocities and propagation modes within these dendrites exhibit anisotropy which is describable by well known relations derived for face-centered-cubic crystal structure; i.e., austenite phase. These variations in wave velocity with crystallographic direction produce large acoustic impedance differences at grain boundaries. Impedance differences also exist near the edges of dendrites due to composition gradients. These gradients forin during cooling when alloy content changes as lower melting point microconstituents form the dendrites and higher melting point microconstituents occupy the volume between dendrites.

A substontial portion of the ultrasonic beam is reflected by the local impedance changes at grain boundaries. These reflections develop signal responses often described as "grain noise", and this "grain noise" is frequently as pronounced as echoes from large cross sectional flaws within the weld zone for austenitic stainless steel. (3) correspondingly, grain noise may be reported as defects, while actual defects may be difficult to locate during inspection. This is energy reflected from defects is often not directed towards the discrete location of transducers. Further, cracks at interfaces between dendrites may be missed because the transmit beam is often collimated along the principal axis of the grains; i.e., a light pipe effect.

Many methods can be used to enhance defect signals and suppress grain noise for austenitic stainless steel weld inspection. Inspecting at lower frequencies reduces grain noise. (4) Grain scatter is less pronounced at lower frequencies where wavelength is much greater than grain size; however, sensitivity to small defects is also reduced. It is often a necessary and laborious step to select a frequency range high enough to see small defects yet low enough to minimize grain noise. Also, the orientation of dendrites is known to have the principal axis pointing towards the center of the weld zone. Thus, there may be propagation directions along which grain scatter is minimal. (5) All these approaches are useful, but all grains are not found to be the same size nor are they aligned in the same direction. Instead, the weld zone is a mixture of size, shapes, and preferred orientations of many microconstituents. 
If the test is tailored to the nominal microstructure, then critical flaws may be missed where microstructure variations are severe. If broad weld inspection standards are to be set and met in practice, the evaluation method should be as microstructurally insensitive as practical. A significant improviment in tnis area has been made, and is the subject of this paper.

\section{DATA ACQUISITION AND ANALYSIS SYSTEM}

An ultrasonic automatic data processing system (UT/ADP) was developed and used to objectively gather waveforms for analysis. The UT/ADP system is illustrated in Figure 1 , and was designed to permit mechanical replication of scans by accurate computer control of transducer positions. In addition, we have ability to retrieve previous scans from stored data files, thus various analys is schemes can be exercised on the same set of waveform data. The UT/ADP system is mini-computer based and includes:

(1) positional I/0 information for the mechanica? test apparatus,

(2) ultrasonic signal capture, digitizing, and mass storage,

(3) numerical analysis and software development capability,

(4) line printer and graphic output, and

(5) operator interface.

This ability to repeatedly reconstruct the original waves permits direct comparison of the analysis methods being developed. Further, these archival data files permit re-interrogation of previously examined samples, without

: the necessity of repeating the tests. These system characteristics which will permit the re-evaluation (or more detailed evaluation) of previously inspected areas. Direct comparison of data obtained from the same sample, but at different times, may be made on any basis from the original RF signal through the most recently developed analytical process. This is an extremely important feature for preserving data from a baseline or preservice inspection, and for later comparison with in-service inspection data.

Special features were included in the UT/ADP system which enables this work to be done in a reasonable time frame. A Tektronix $R 7972$ transient 


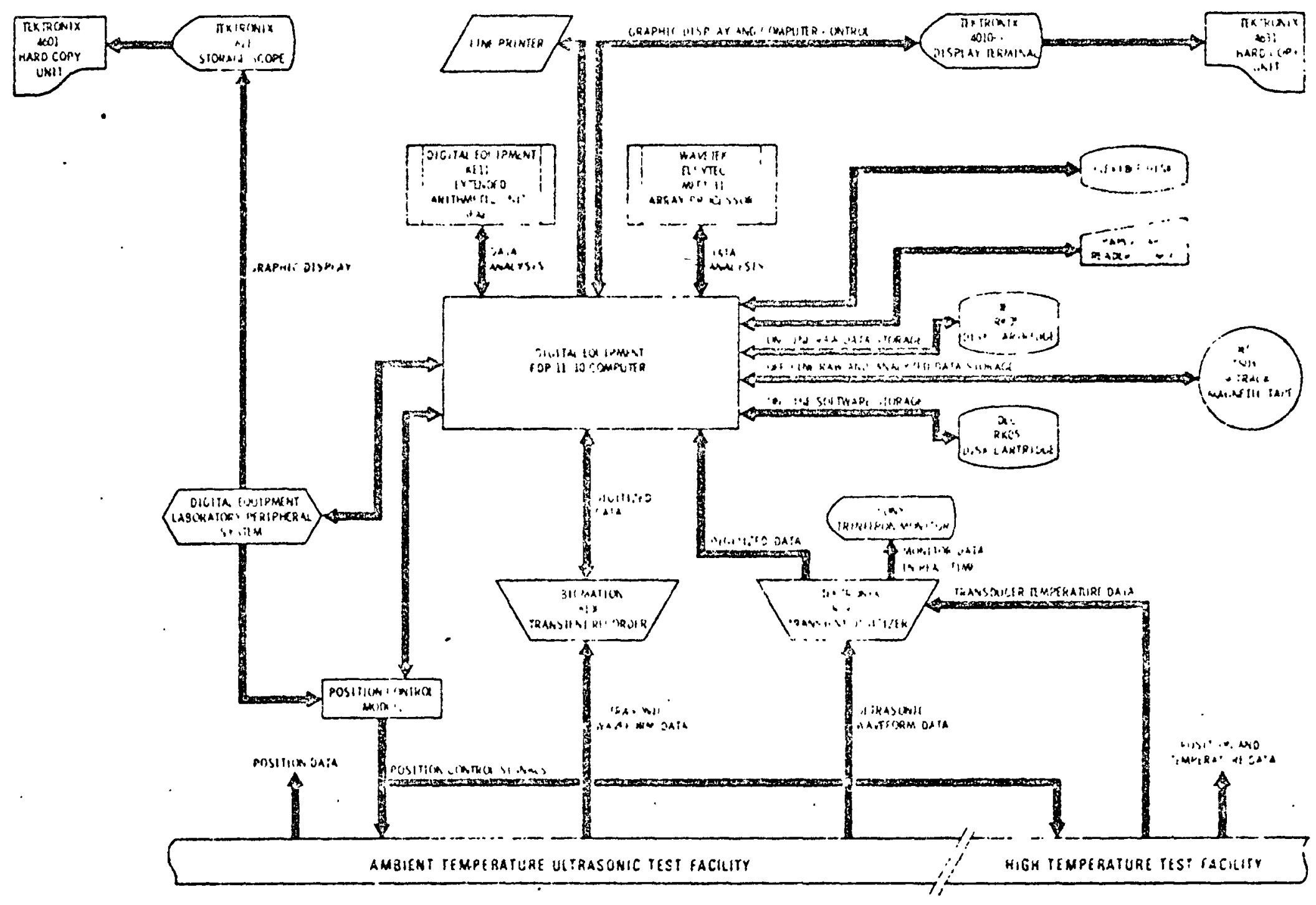

Figure 1. UItrasonic Automatic Data Processing System 
digitizer is employed to capture and digitize ultrasonic waveforms. The Tektronix Scientific Programming System (SPS), a BASIC language software system, permitted efficient development and testing of the various numerica? analysis algorithms, and an Elsytec array processor, which enables the UT/ADP system to analyze large numbers of data in a short period of time.

\section{SPECIMEN AND DATA ACQUISITION TECHNIQUES}

An austenitic stainless steel pipe section, 8-inch diameter schedule 40, was notched at several locations to simulate defects in the weld. As illustrated in Figure 2, these notches were electro-discharged machined (EDM) $25 \%$ and $50 \%$ through the wall thickness at three orientations. The notched pipe was scanned using longitudinal and shear immersion inspection methods. The transducer was a $3 \mathrm{MHz}$, $80 \%$ bandwidth, dual element unit, (1/4" $\times 1 / 4^{\prime \prime}$ square) and it was designed to operate in a side-by-side pitch-catch mode. The angle between transmit and receive beams was approximately $10^{\circ}$ in stainless steel. This technique is similar to those used by others for austenitic stainless steel weld inspection. $(6,7)$

Scanning was accomplished by rotating the pipe at about 30 RFM with the transducer fixed in position. There was no longitudinal advancement of the transducer used in this study. A precision encoder was used to input rotational position to one counter. A second counter was advanceable via computer control, and the transmit pulse was strobed when the two counter registers agreed. By advancing the second counter, 500 waveforms were digitized and stored for one scan around the tube. Each waveform was stored as 512 words ( 16 bits each) corresponding to a 20 microsecond record length.

The beam path is illustrated in Figure 2, and was aligned to give peak return from the large center weld notch; i.e., type $c-50 \%$. Time on the digitized records corresponded to position from the inner surface through the weld zone to the outer surface. 

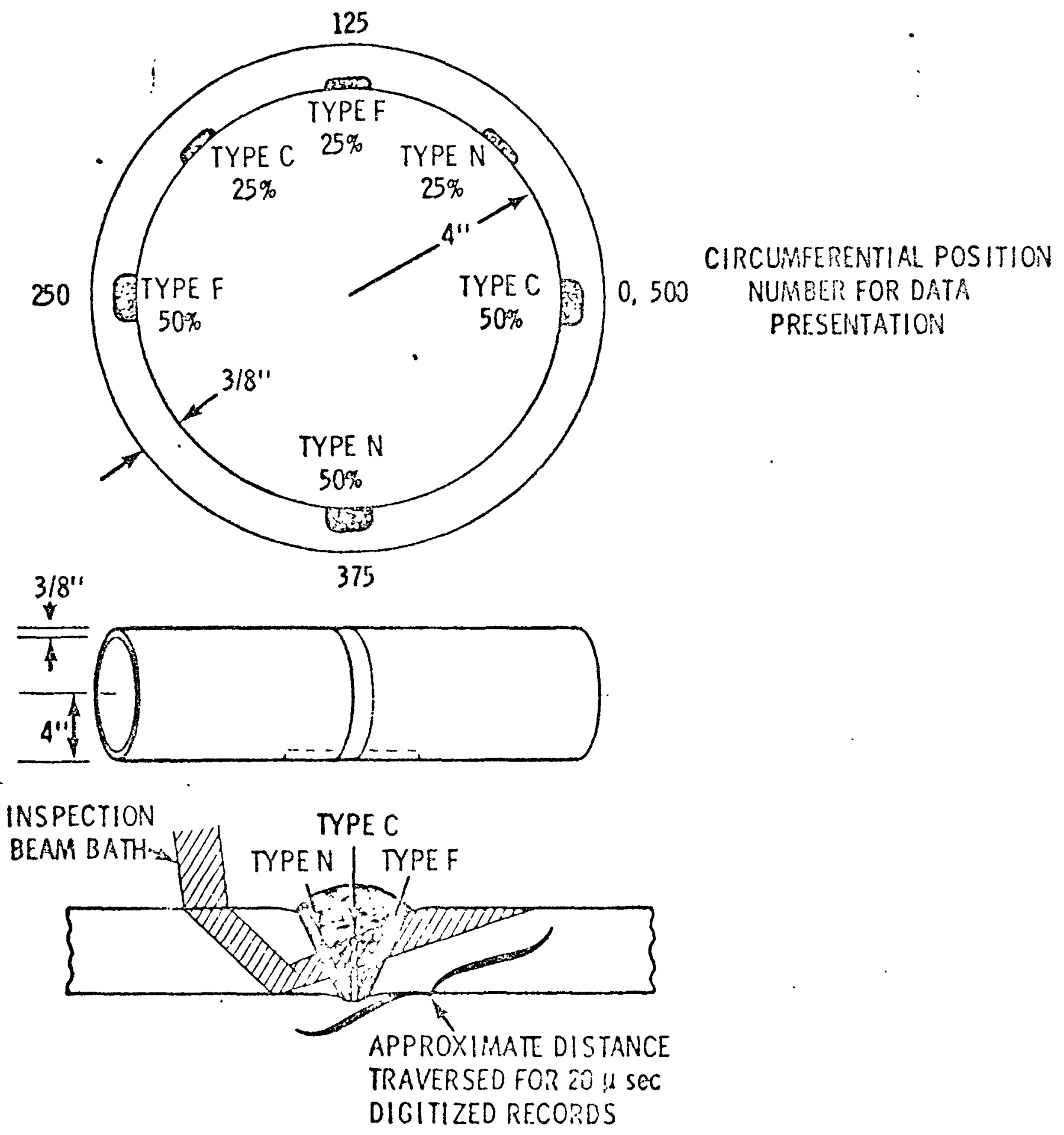

Figure 2. Inspection Beam Path and Notch Locations 
DATA ANALYSIS TECHNIQUES

Digitized time domain waveforms were processed by three computational methods. These were by detecting amplitude, by cross-correlating to a waveform from a known broadband discontinuity, and by transforming to the frequency domain using a fast fourier transform (FFT) algorithm.

\section{Peak Amplitude}

Time doma in amplitudes were computed using:

$$
v_{i}=\max \left\{\left|v_{j}\right|: j=4 i-3, \ldots ., 4 i\right\}
$$

where $i=1, . ., 128 ; V$ is the amplitude waveform and $V_{i}$ replaces four data points of the RF waveform $v_{j}$ by the peak amplitude in the internal $\{j=4 i-3, . ., 4 i\}$. This calculation reduces the number of points per record from 512 to 128 , which reduces storage requirements when only detected amplitude results are desired.

Equation (1) has the effect of full-ivave rectifying and smoothing the RF waveform as illustrated in Figure 3. This was developed to produce a result suitable for isonetric plots which are presented later. It is similar to amplitude detection performed with conventional UT hardware.

\section{Cross-Correlation}

The cross-correlation algorithm may be used to determine where two waveforms agree or align in detail. $(8,9)$ specifically, it can be used to determine if, or where, a known echo pattern is contained in a noisy waveform record. Cross-correlation is useful for two reasons. First, it can be used to produce a time domain plot of echo amplitudes versus time which contains less clutter than the result of Equation (1), and this will be demonstrated in this study. Second, cross-correlation can be used to indicate where echo activity is in the time domain, which may then be used for the purpose of 


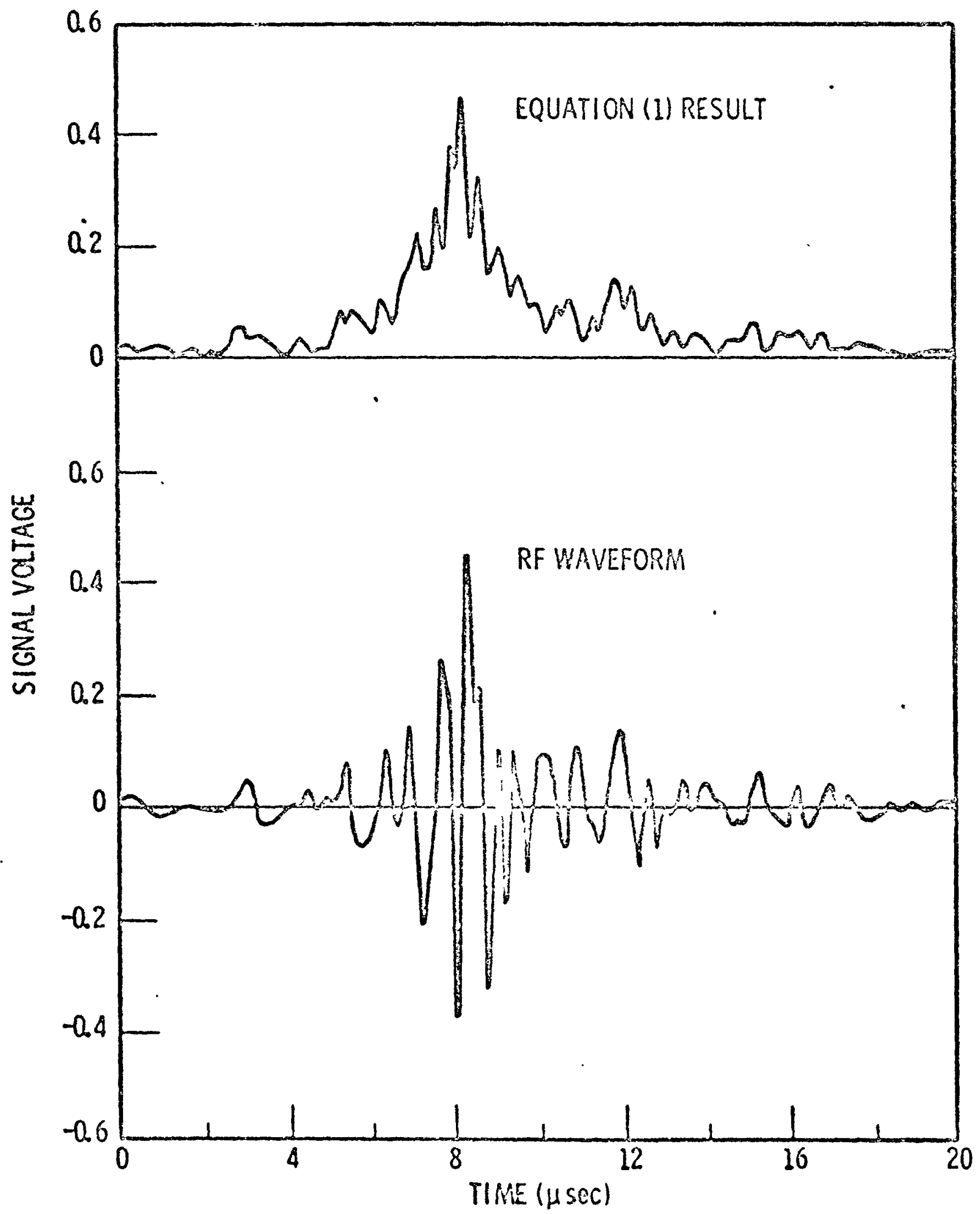

Figure 3. Example of Peak Amplitude Detection 
setting waveform gate times. This second aspect was not used in this study but is expected to be an important step in analysis in the future.

We compute cross-correlation according to:

$$
W_{k}(A, B)=\frac{\sum_{\substack{i, j=1 \\ N}}^{N} A_{i} B_{j} \delta_{i, j+k}}{i, j=1}
$$

Here $\delta$ is the Kronecker delta defined as:

$$
\begin{aligned}
& \delta_{m n}=1 \text { for } m=n, \\
& \delta_{m n}=0 \text { for } m \neq n,
\end{aligned}
$$

$N=512$ is the number of points in the digitized waveform, $W$ is the crosscorrelation results, and $k$ is an offset position index. This offset corresponds to sliding waveform $B$ past waveform $A$, and peaks in $W$ result for offsets where $A$ and $B$ align.

For analysis here, $B$ was the echo from a back corner reflection from a calibration block, and A represents received RF waveforms. Typical results are shown in Figure 4. The position of the peak indicates where each pair aligns best. If more than one peak occurs in the cross-correlation result, these peaks indicate the probable echo locations on the original waveform.

For plotting purposes, a peak amplitude form of $W$ is useful. This was computed using a procedure similar to that used for Equation (1):

$$
w_{\ell}(A, B)=\max \left\{\left|W_{j}(A, B)\right|: j=4-3, \ldots, 4 \ell\right\},
$$

with $\ell=-64, \ldots, 64$; and $w$ is peak amplitude result of the crosscorrelation function. When $\&$ ranges from -64 to 64 , offset time ranges from $-10 \mu \mathrm{sec}$ to $10 \mu \mathrm{sec}$. 


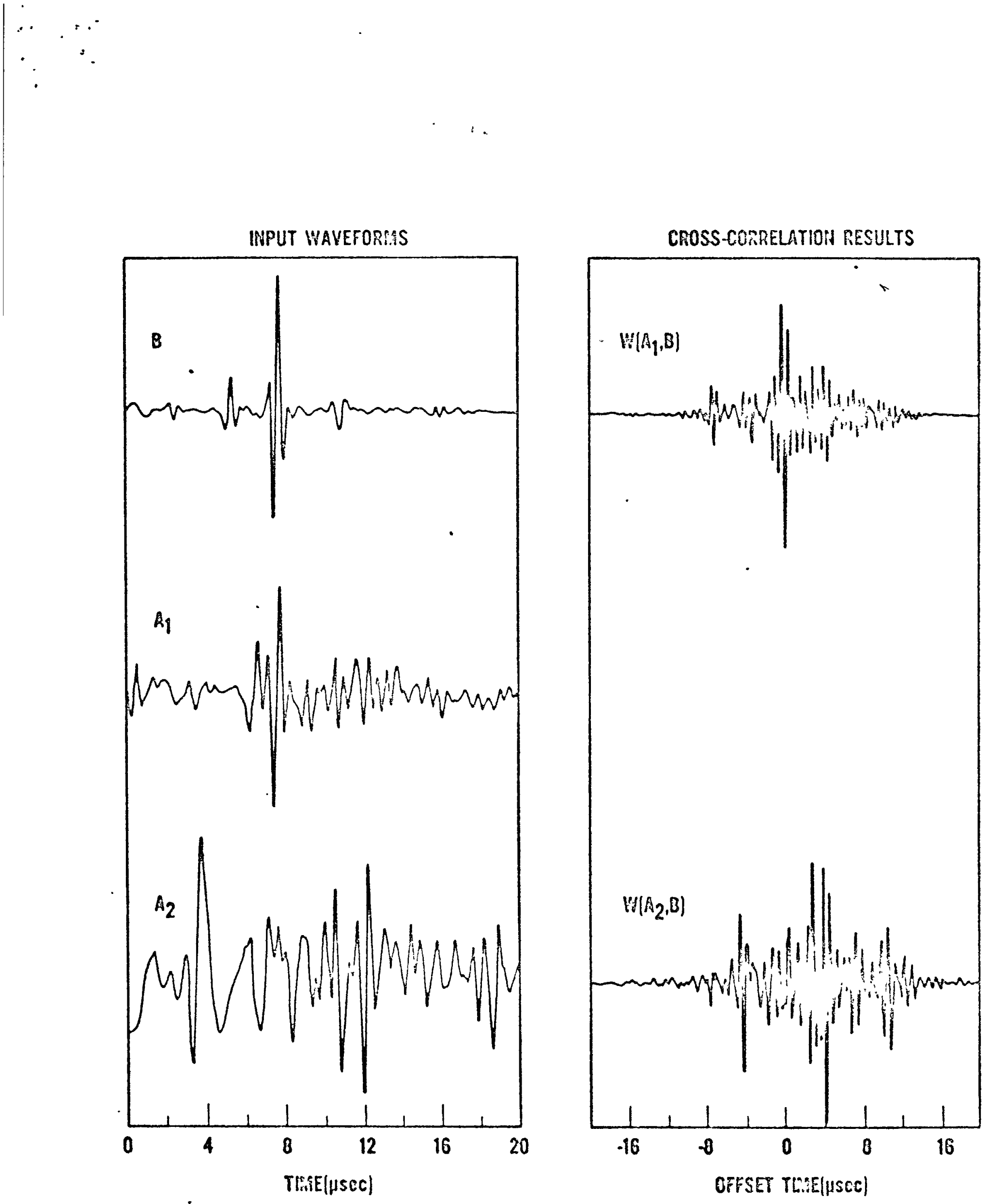

Figure 4. Example Cross-Correlation Results. 


\section{Frequency Domain Analysis}

The FFT algorithm was used to analyze the frequency content of received waveforms. (10) From this algorithm, coefficients $A_{\omega}$ and $B_{\omega}$ are related to the time domain waveform by:

$$
f(t)=\int_{0}^{\infty}\left(A_{\omega} \cos \omega t+B_{\omega} \sin \omega t\right) d t
$$

and the power spectral density is:

$$
p(\omega)=\left(A_{\omega}^{2}+B_{\omega}^{2}\right)^{1 / 2}
$$

Simple moments are useful for comparing frequency distributions:

$$
\begin{aligned}
M_{0} & =\int_{0}^{\infty} p(\omega) d \omega \\
M_{1} & =\int_{0}^{\infty} p(\omega) d \omega \\
M_{2} & =\int_{0}^{\infty} \omega^{2} p(\omega) d \omega
\end{aligned}
$$

The zeroth moment, $M_{0}$, is equivalent to the total power. Futher, by differentiating (5) with respect to time, one can show that $M_{1}$ and $M_{2}$ are related to the total power of the first and second derivative with respect to time of the original time domain waveform. This is also a standard deviation theorem for fourier transforms. (11)

We found the cumulative definite integral with variable upper limit, $\omega$, useful for detailed study of the frequency patterns from defects. This may be expressed as:

$$
M_{k}(\omega)=\int_{0}^{\omega} \xi^{k} p(\xi) d \xi
$$

in discrete form this becomes: 


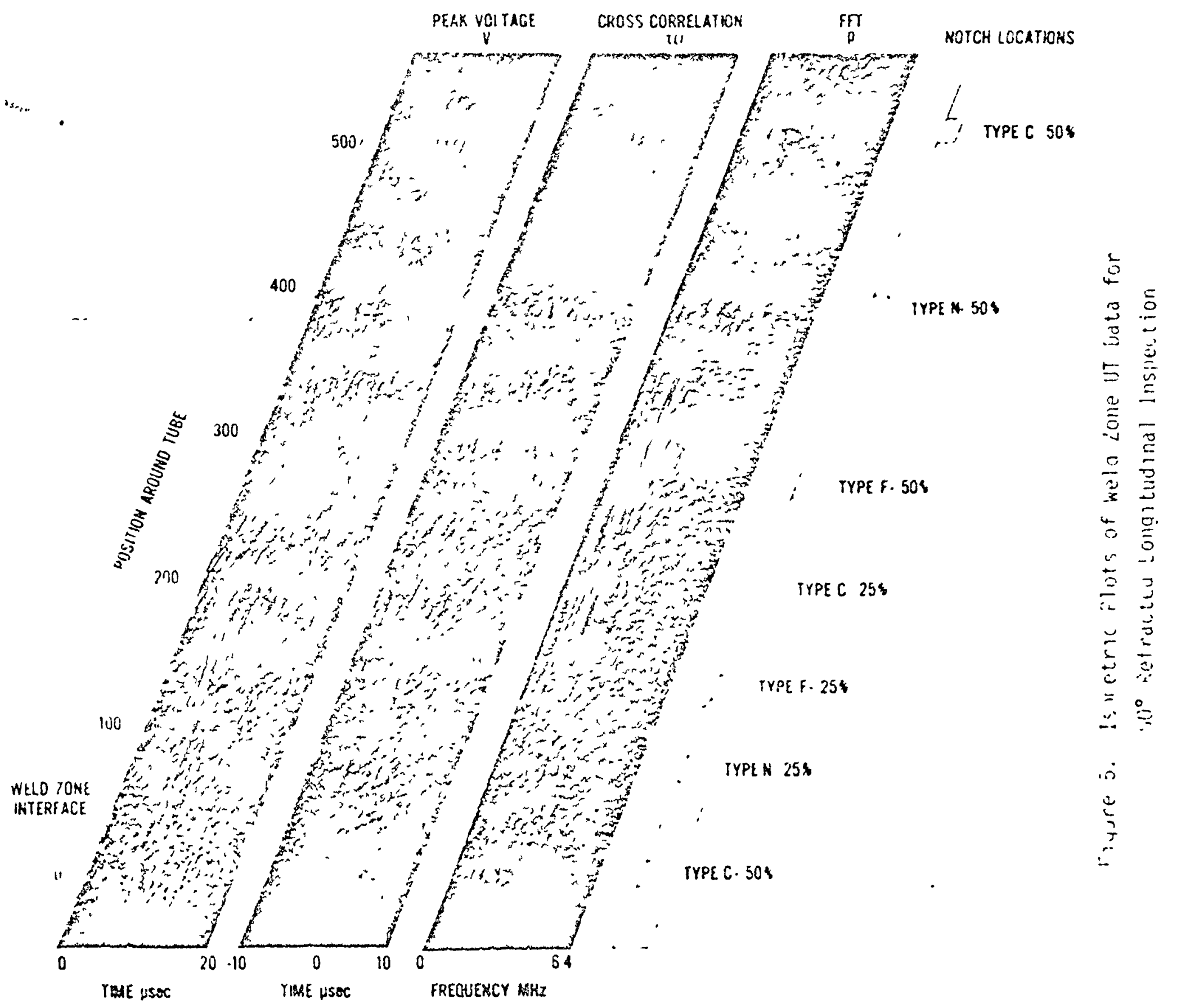




$$
M_{k}(i)=\sum_{j=1}^{i} j^{k} p_{j}
$$

where $i$ is a frequency index and $p_{j}$ is the discrete analog of Equation (6). Plots of $M_{0}(\omega)$ and $M_{2}(\omega)$ are presented in Figure 6 and are discussed in the next section.

\section{Adaptive Learning Network}

An Adaptive Learning Network (ALN) was implemented similar to that described by Mucciardi. (1) This process consists of a network of multinomial combinations with each combination $\hat{y}$ computed for two imputs $x_{i}$ and $x_{j}$ as:

$$
\hat{y}=a_{1}+a_{2} x_{i}+a_{3} x_{j}+a_{4} x_{i}^{2}+a_{5} x_{i} x_{j}+a_{6} x_{j}{ }^{2}
$$

The coefficients and specific network construction are determined by a training process described later. Refer to Figure 7 for a typical network.

Once the training process has been completed, that is, the coefficients and structure have been determined, the network serves as a model with vector inputs $x_{i}$ from ultrasonic test data. Model output could be defect existence, type, or size depending on the particulars of the training process.

Before the training process begins, the data are grouped into three sets; a training set, a testing set, and a verification set. During the training process, all possible pairs of vectors from the training set are combined as shown above. For each combination, the coefficients are determined so as to minimize mean square error between calculated output, $\hat{y}$, and the desired output, $y$. The testing set is used to see if the fit is a good one; i.e., error between $\hat{y}$ from the testing set and $y$ is not significantly larger than the training error. If the fit is not good, the combination is rejected and the next pair is considered.

In this manner, a new group of vectors is generated from the best combinations of the original vectors. The process can be continued to as many 
CUAULATIVE HOSEKT

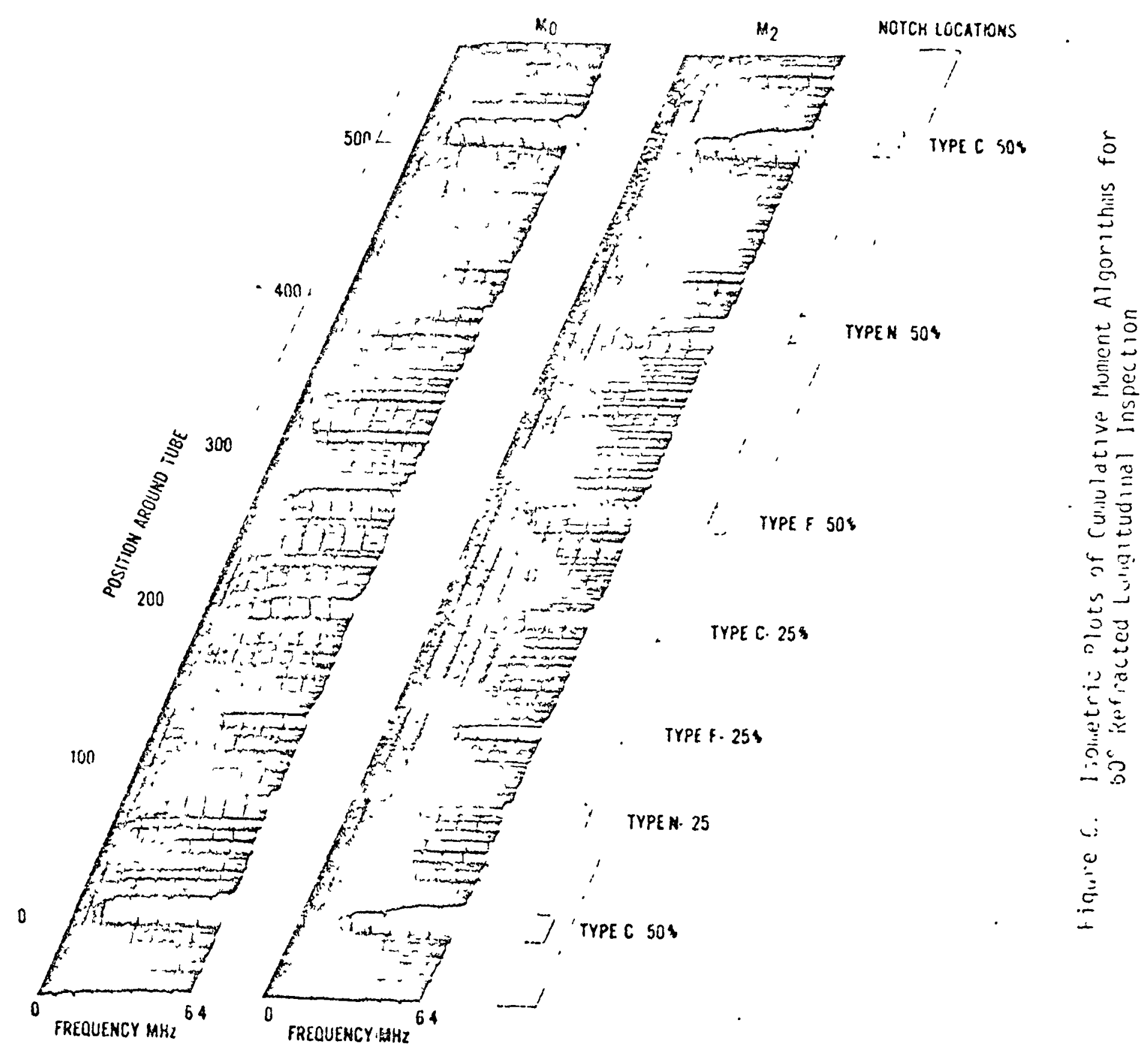




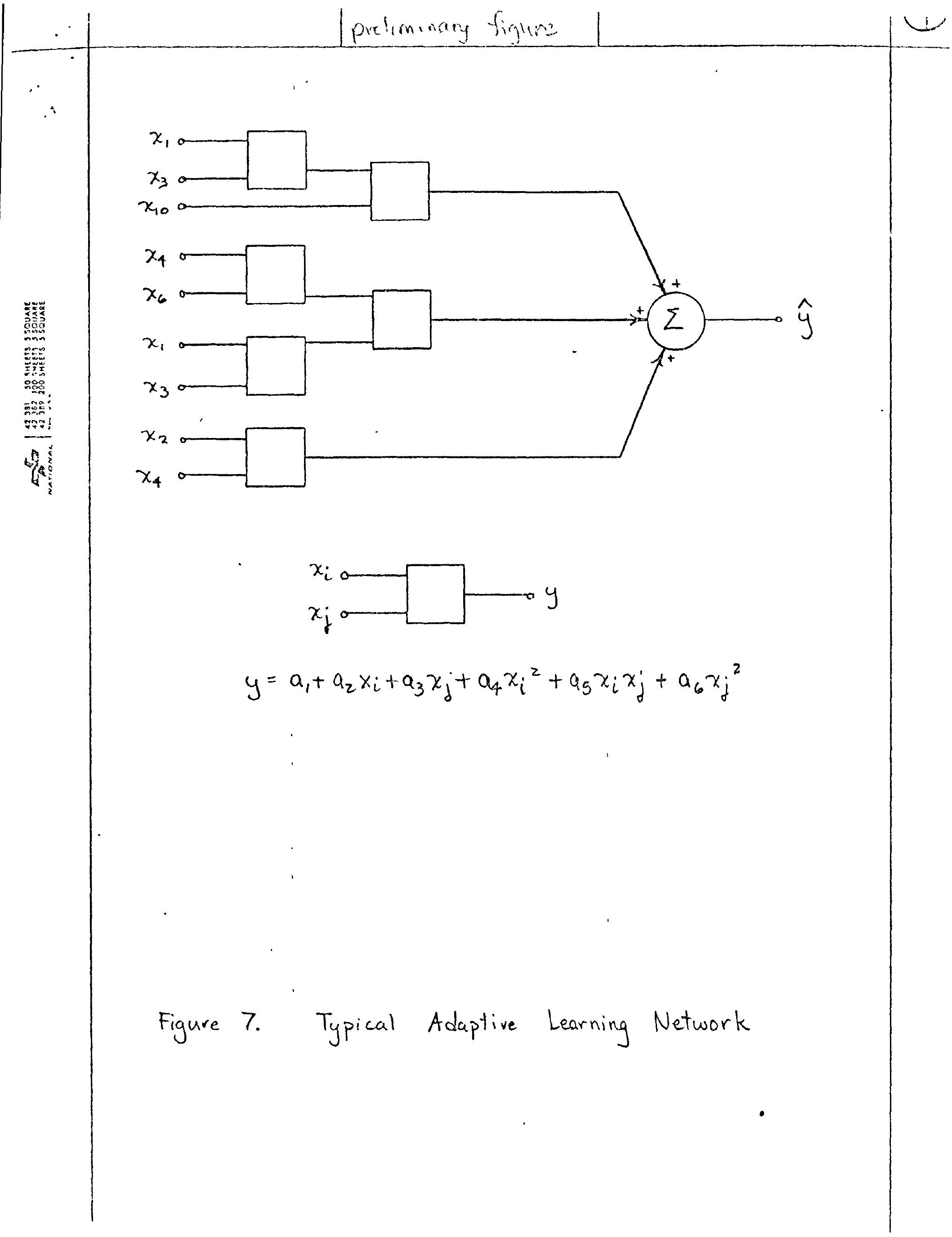


levels as desired - usually until no improvement is shown. The best vectors in the final level can then be averaged to form the overall network. After the final network has been configured, the verification set is used to estimate expected accuracy.

The above training process was implemented using data from the refracted longitudinal scan discussed previously. Actual vector inputs selected were:

(1) Peak voltage (5-15 usec time interval)

(2) Peak voltage $(0-5,15-20 \mu \mathrm{sec}$ time intervals)

(3) Peak voltage $(0-20 \mu \mathrm{sec}$ time interval)

(4) Vector $1 /$ vector 2

(5) Peak cross-correlation (5-15 $\mu \mathrm{sec}$ time interval)

(6) Peak cross-correlation $(0-5,15-20 \mu \mathrm{sec}$ time intervals)

(7) Peak cross-correlation $(0-20 \mu \mathrm{sec}$ time interval)

(8) Vector 5/ Vector 6

(9) Frequency response maximum

(10) Frequency of the maximum

(11) $M_{0}(6.4 \mathrm{lhz})$ (see equation 10)

(12) w: $M_{0}(w)=.2\left(M_{0}(6.4 \mathrm{Mhz})\right)$

(13) $\omega: M_{0}(\omega)=.4\left(M_{0}(6.4 \mathrm{Mhz})\right)$

(14) $\omega: M_{0}(\omega)=.6\left(M_{0}(6.4 M h z)\right)$

(15) $w: M_{0}(w)=.8\left(M_{0}(6.4 M h z)\right)$

(16) $M_{2}(6.4 M h z)$ (see equation 10)

(17) $\omega: M_{2}(\omega)=.2\left(M_{2}(6.4 M h z)\right)$

(18) $\omega: M_{2}(\omega)=.4\left(M_{2}(6.4 M h z)\right)$

(19) $\omega: M_{2}(\omega)=.6\left(M_{2}(6.4 M h z)\right)$

(20) $\omega: M_{2}(\omega)=.8\left(M_{2}(6.4 M h z)\right)$

\section{RESULTS}

Isometric plots of detected waveform amplitude from Equation (1), cross-correlation algorithm results from Equation (4), and frequency domain power spectral density from Equation (6) are compared in Figure 5 for the 
$60^{\circ}$ refracted longitudinal scan. The peak voltage and cross-correlation results are presented here for about the same time domain window, and this window, $20 \mu \mathrm{sec}$ wide, corresponds to a propagation path from the back surface through parent material, across the weld zone boundary, and through the weld zone to the top surface of the specimen as illustrated in Figure 2 . The weld zone interface is visible as a ridge on the time domain records, and note that the ID surface echo is visible from circunferential position 190 through 310. The pipe was not perfectly round, and this caused the skew as the water path distance increased from 190 to 310 .

The time domain plot from the cross-correlation algorithm contains less clutter than the peak voltage plot. Particularly, note the enhanced detail of the notches as compared to the ID surface echo and weld zone interface echo. Also, we find the notch type (near side, center, or far side) can be confirmed by noting the location in time of signal peaks with respect to the ridge from the weld zone interface.

The frequency domain data presented in Figure 5 show a broadband response is received at each of the notch locations. We find this broadband response is typical of clean or distinct echos. Note the interference structure that is visible as frequency domain peaks in the vicinity of the larger notches. Although this structure was not analyzed in this study, we expect it should be predictable from the notch angle and type as has been demonstrated by other investigators. $(12,13)$

The attenuation influence of the weld zone is also visible from the frequency domain data plot. Specifically, near side notches show more information than do far side notches. This is expected because attenuation of the weld zone is progressively more severe at higher frequencies, and the wave path to far side notches traverses the most weld zone material.

Moment results are presented in Figure 6. These computations are useful to show how energy is distributed in the frequency domain. First order 
moment, $M_{0}$, is cumulative power and a normalized form of this function has been an important vector input to the adaptive learning network of Adaptronics. (1) We favor the second order moment, $M_{2}$, because it appears sensitive to the broadband signals reflected from notch type defects, and is less sensitive to "grain noise" which we find is more narrow banded. Hopefully, future studies will establish that cracks, like notches, are more broadband reflectors.

To the extreme right on the cumulative moment plots; i.e., at $6.4 \mathrm{MHz}$, peaks correspond to notch locations. These moment results are compared to peak voltage and peak cross-correlation results in Figure 8. If data from the total $20 \mu \mathrm{sec}$ window is used, the second order moment, $M_{2}$, yields superior results insofar as locating notches. Peak voltage is least accurate, and peak cross-correlation and zeroth order moment, $M_{0}$, show fair sensitivity to notch locations.

Peak voltage and peak cross-correlation results are also shown in Figure 8 as recomputed for a reduced time interval, 7.5 to $15 \mu \mathrm{sec}$, so as to avoid the back surface echo and to concentrate more on signals within the weld zone. We find the signal-to-noise ratio is still not as good as second order moment computed over the entire $20 \mu \mathrm{sec}$ record. This is because a voltage peak can occur and will be reported in the time domain regardless of the frequency of the signal and many of these peaks are from narrow band signals, whereas, the second order monent is more sensitive to broadband signals.

Radiographic analysis from this pipe is compared to second order moment results in Figure 9. Most all "non-notch" peaks were found to be associated with slight porosity within the weld zone. We found qualitative analys is of the "non-notch" peak near circumferential position 50 interesting. From data of Figure 8, it is difficult to deduce any distinguishing features of this signal peak which might be different from the notches. However, detailed examination of time domain data isometric prints, Figure 5 , suggest this signal source is in the center of the weld zone. This is because the peak lies well behind the interface ridge but ahead of the far side notches. Analys is of the frequency isometric print shows a narrow band of high frequencies reflected. This is different than is exhibited for the notches. All these observations are consistent with the porosity reported from radiographic analysis. 
$\therefore \quad$ Insert (4) before this page

PEAK VOLTAGE

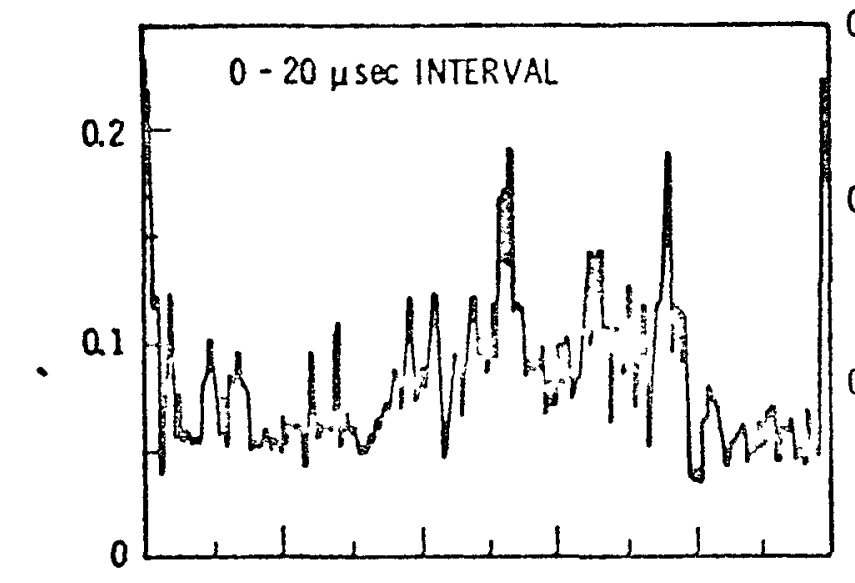

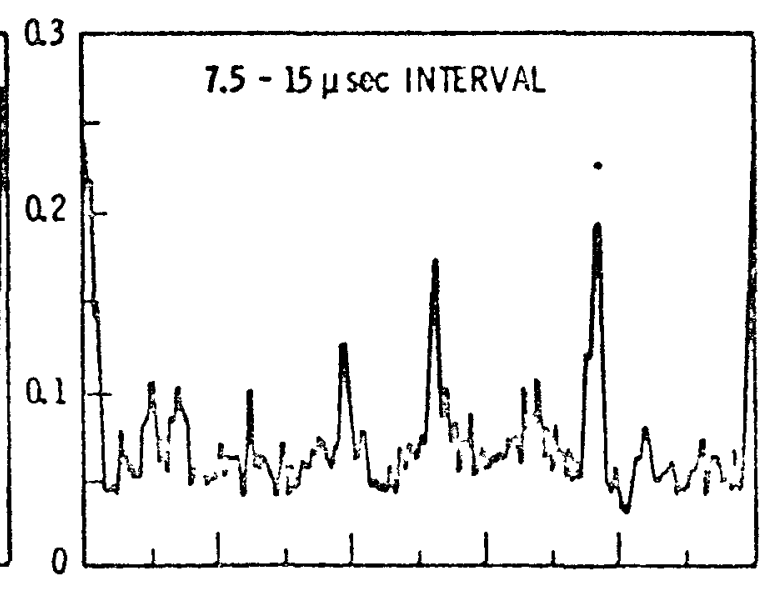

PEAK CROSS-CORAELATIO:
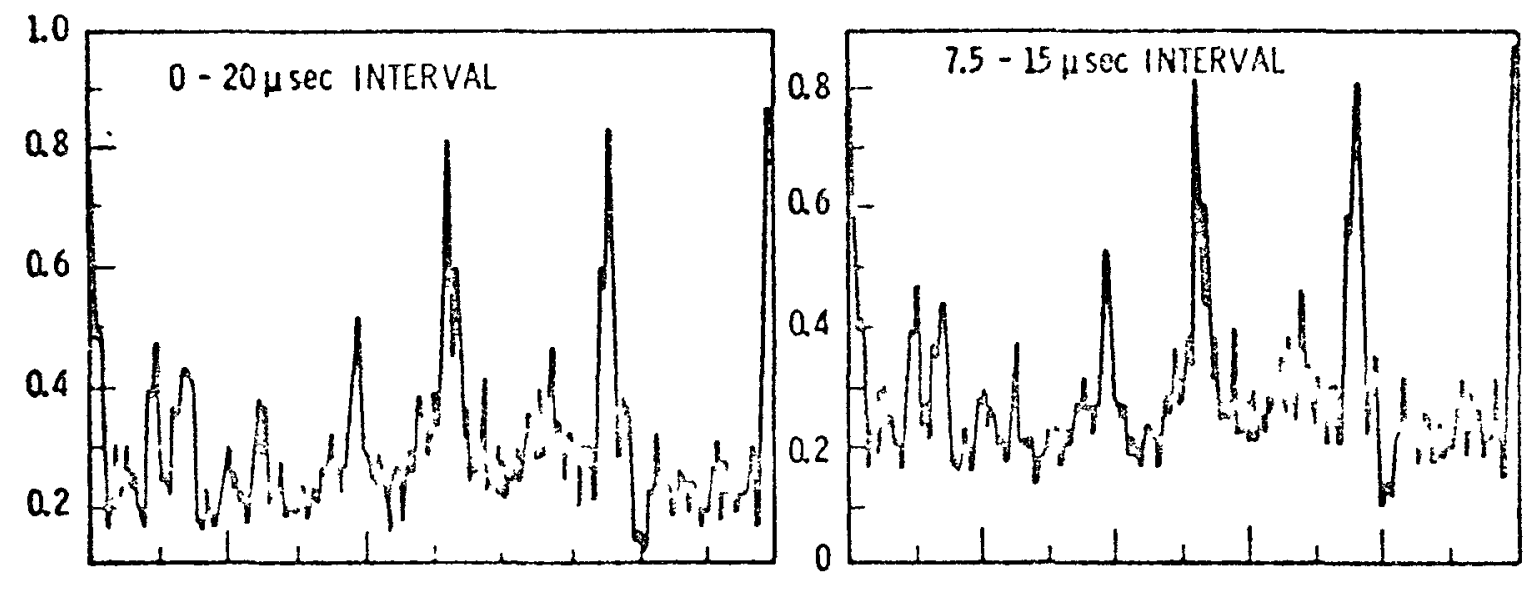

MOREET ALGOZITIMSS

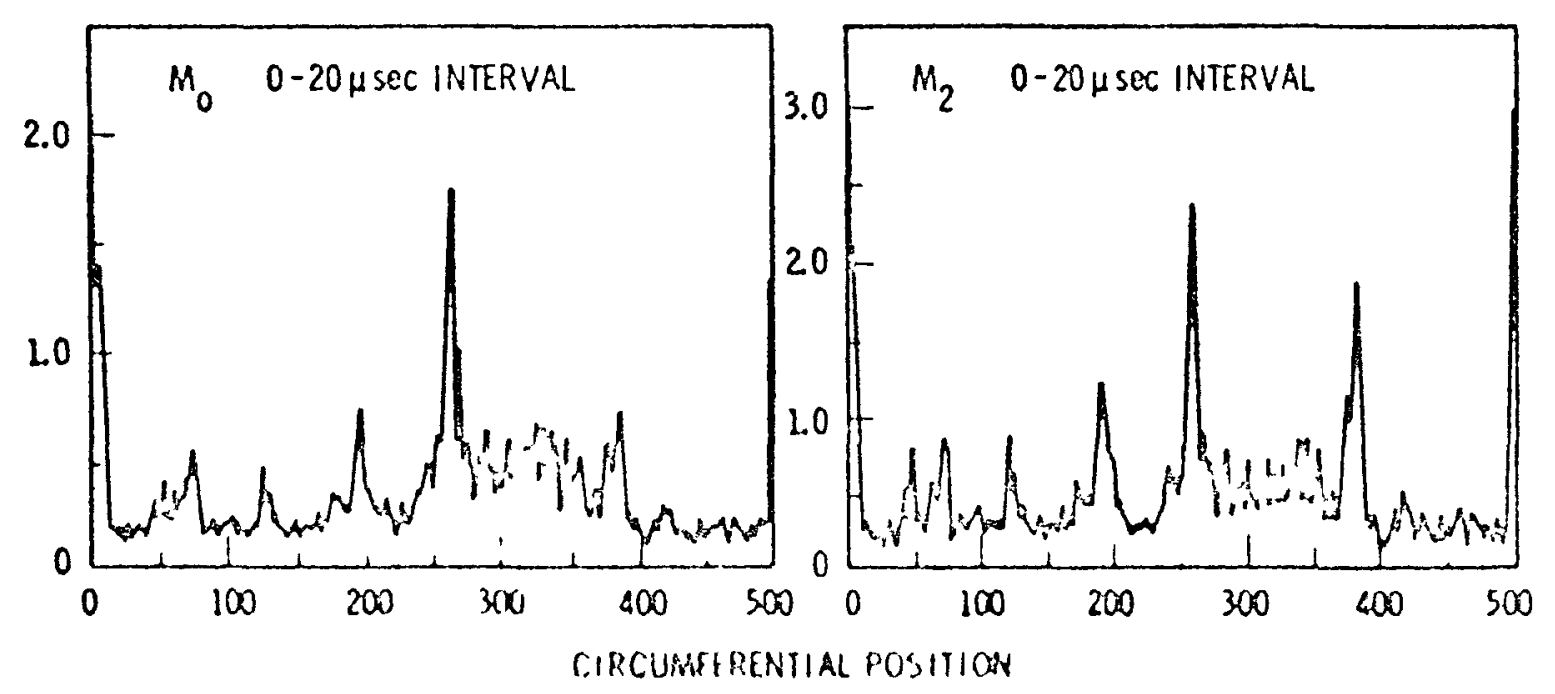

Figure $\gamma^{\prime} B \begin{aligned} & \text { Peak Voltage, Total Power } M \text {, and Moment } M_{2} \text { tor } 60^{\circ} \\ & \text { Refracted Longitudinal Inspection }\end{aligned}$ 


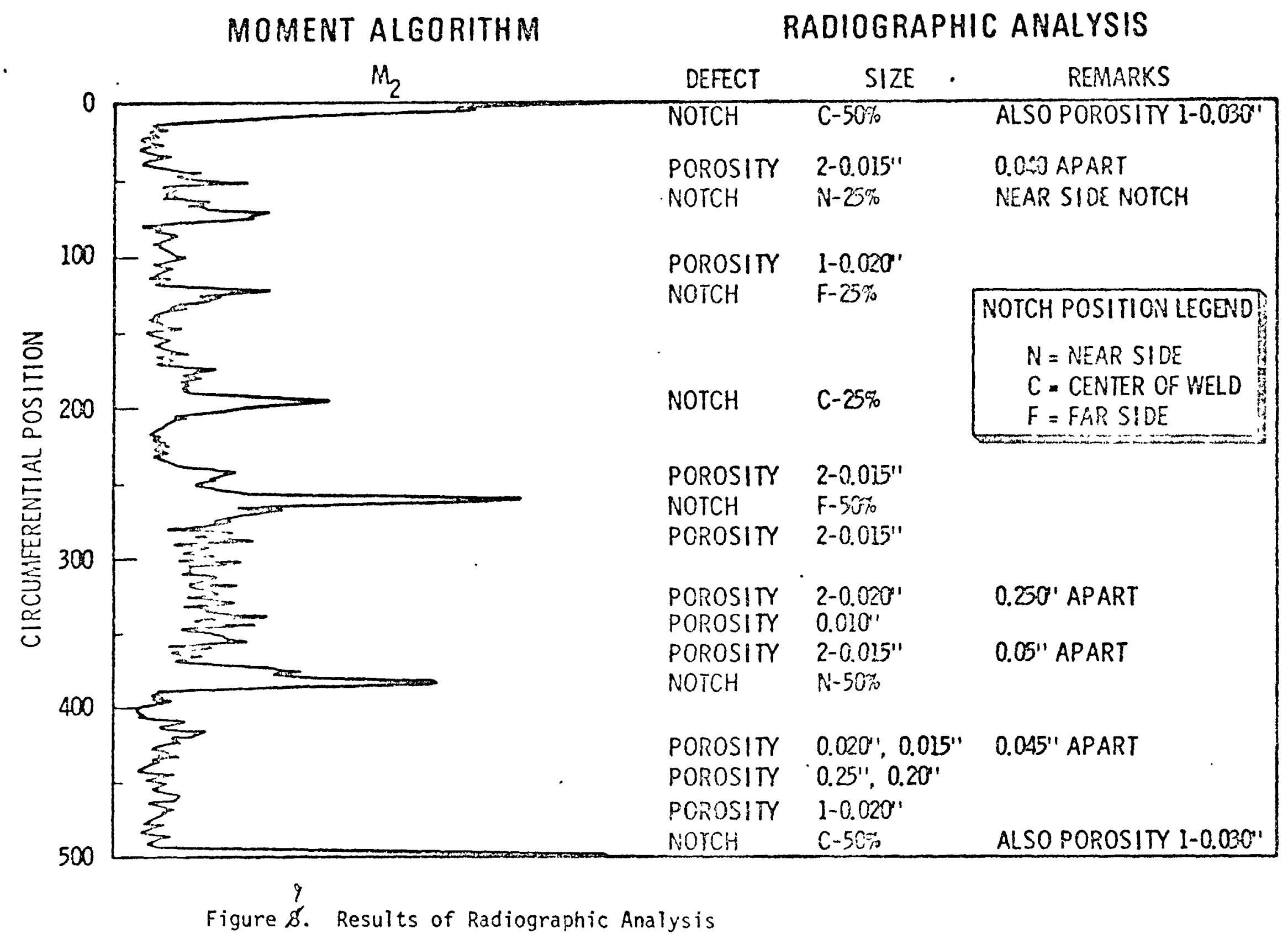


As a further example of using the second order moment, a $45^{\circ}$ shear wave inspection scan was made using the same transducer. Results are compared in Figure 10, and we found that the second order moment was sensitive to al1 notches within the $45^{\circ}$ shear wave beam path. Further, porosity peaks again agreed with results from radiographic analysis.

Two networks were created with the ALN process. The first network was trained to detect both notches and porosity while the second network was trained to detect only notches. Refer to Figure 11 for plots of the output of both networks.

In the first network, the two notches that were part of the verification data set were successfully located. The noise level is way down except for several peaks in regions where radiographic analysis showed porosity. In the second network, the training and testing data sets were carefully selected to include regions with porosity. Most of the porosity peaks were successfully damped out. Again, the notches in the verification set were found and the noise level was very low.

\section{CONCLUSIONS}

1. We have found a second order moment algorithm to be useful for discriminating between signals from defect notches in austenitic stainless steel welds and grain noise. This algorithm involves a moment sum of frequency domain data, and gives best results with broadband transducers and electronics. Discrimination against grain noise is good because spectral response of grain noise signals is narrow band, whereas response from defects is broadband.

2. Isometric plots of UT data provide a useful analysis tool for qualitative evaluation of signal patterns. Plots of detected signal voltage and frequency power spectral density complement each other, and can be used to deduce defect locations. 
$\mid \begin{array}{ll}\because & \ddots \\ \because & \ddots \\ \ddots & \end{array}$

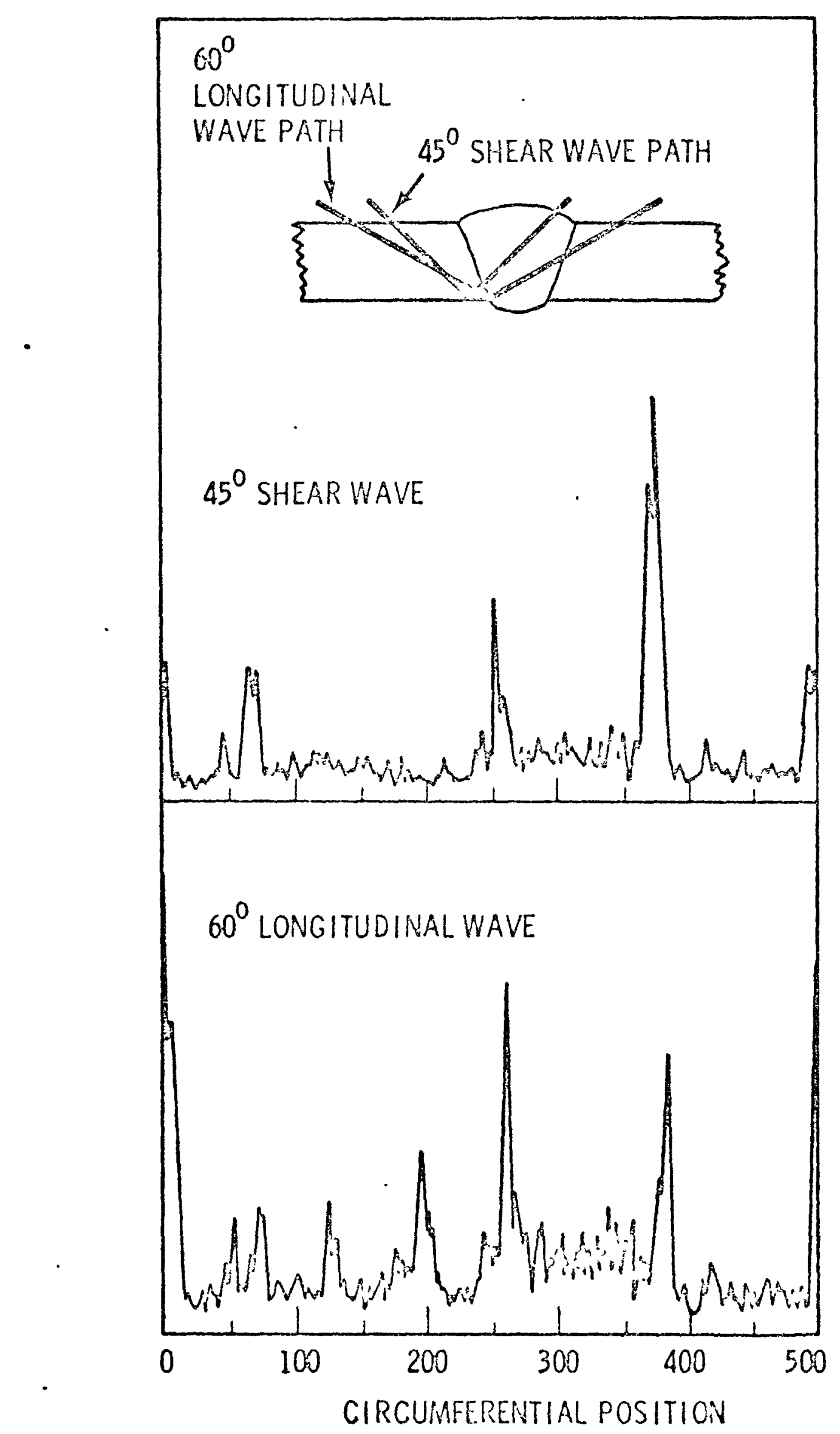

10

Figure 9 . Comparison of moment $H_{2}$ for $60^{\circ}$ rongitudinal and $45^{\circ}$ shear inspections. 


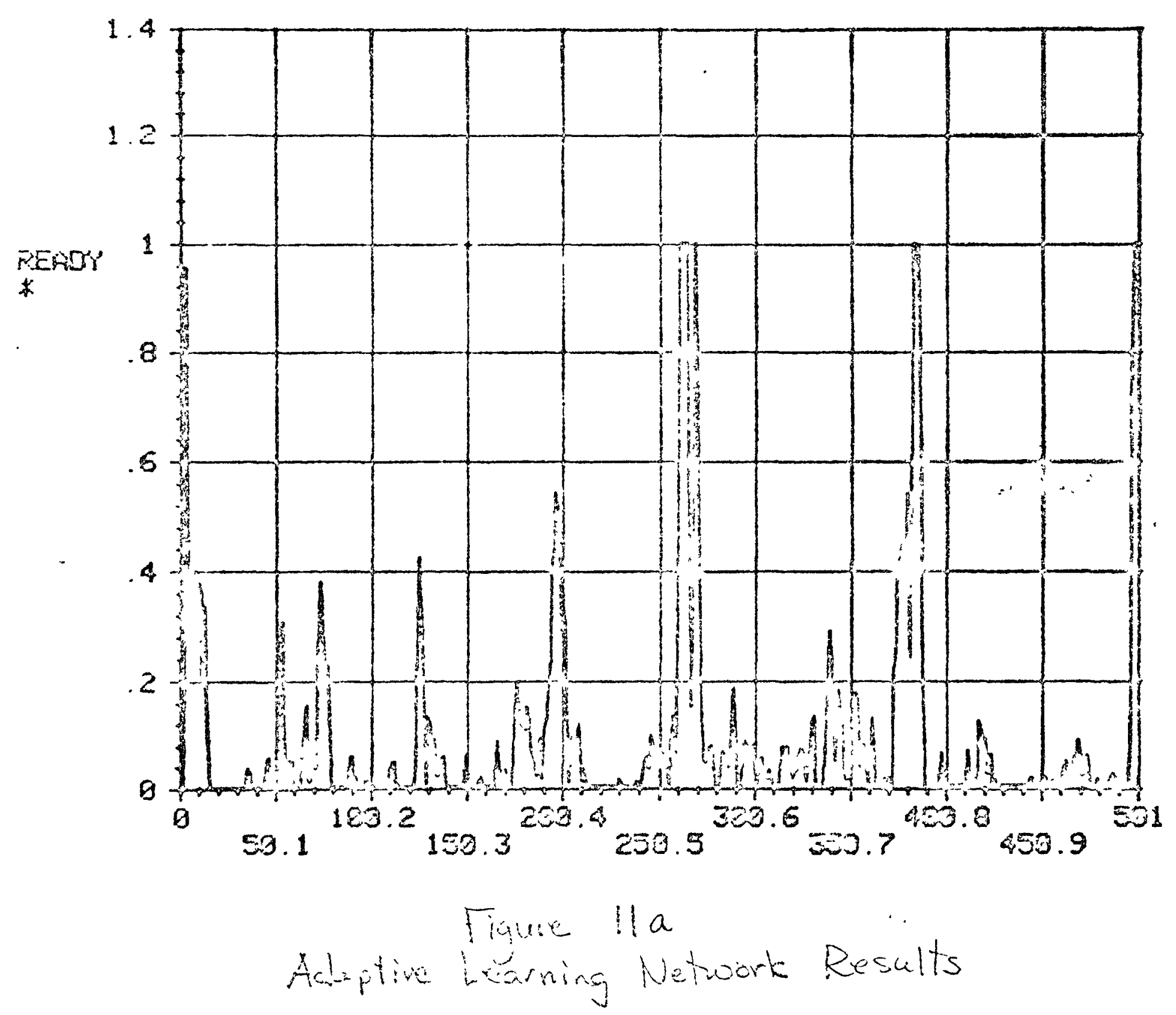




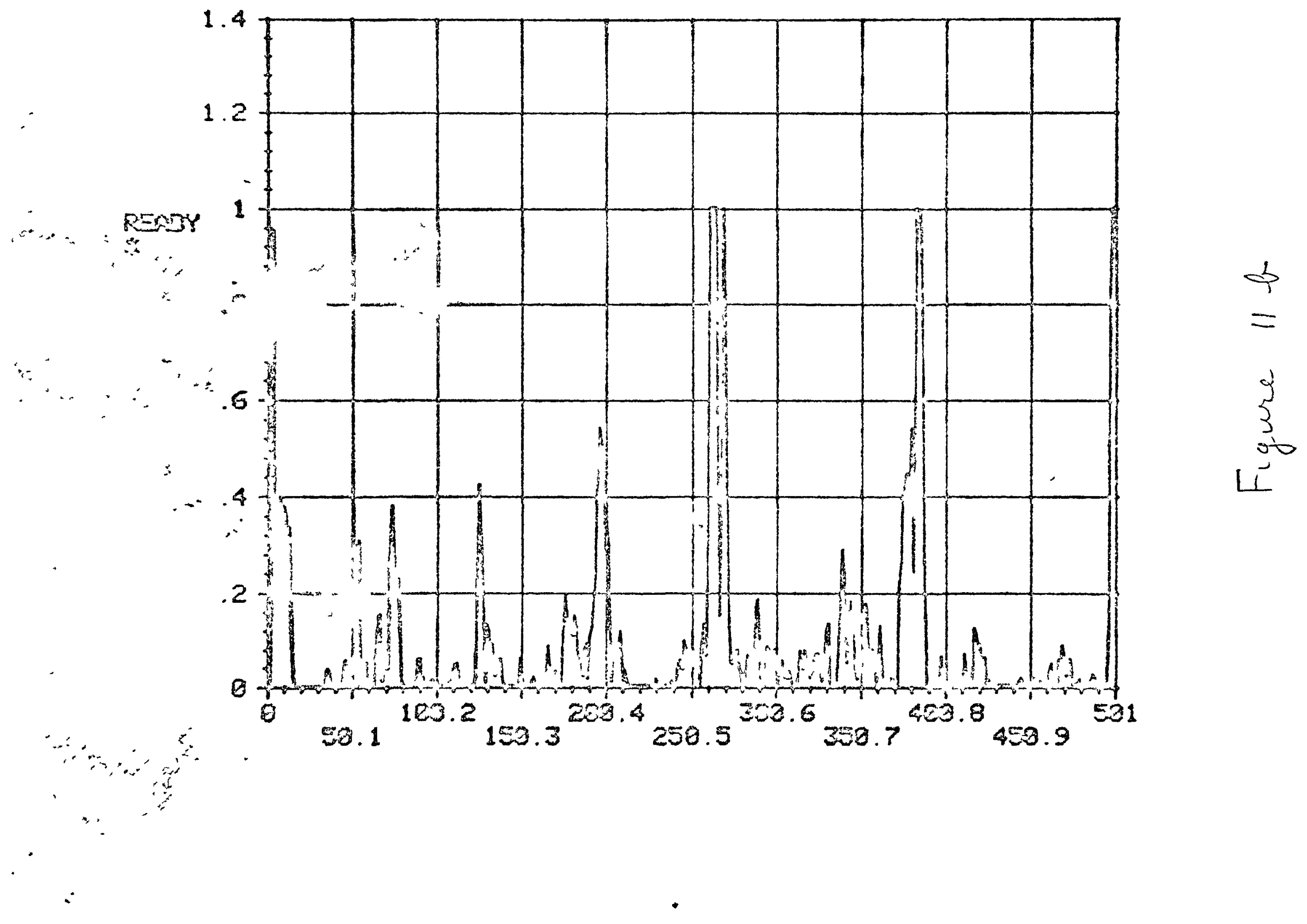


3. A cross-correlation algorithin can be used to determine where or if known echo patterns exist in a time domain record. Plots of peak crosscorrelation results contain the same information as peak voltage results, but have the value of enhancing signals from the notch locations.

4. From even the small number of networks generated thus far, it is evident that ALN techniques show a great deal of potential. We have been successfur in discriminating between notches and porosity, and grain noise has been virtually eliminated.

5. Classical test methods, which compare signal amplitude to that from known reflectors, are not recommended for austenitic stainless steel welds. Peak voltages from "grain scatter" were often as large as signals from defects, and this would result in erroneous defect reports. Further signals from notches were often buried in grain noise, thus, obscured, and these defocts would be missed by classical test methods. It should be realized that our comparison here is for broadband testing. We would expect some improvement in classical signal amplitude correlation with defects if the test were narrow band and in the proper frequency range. Further, the broadband results, specifically the isometric plots of frequency response, would be a proper tool to deduce the appropriate narrow band range to choose.

6. For examination of critical welds, a multi-algorithm inspection scheme is proposed. One algorithm, such as the moment algorithm presented here, would be used to locate areas which are potentialiy defective. Other algorithms, such as frequency analysis, cepstrum analysis, or phase front analysis, would be used to size and provide positive defect identification along with a more detailed interrogation of the suspect areas. 


\section{REFERENCES}

1. Nucciardi, A. N., Chang, J. K., and Lawrie, W. E., "Development of Adaptive Learning Networks for Pipe Inspection, Task I, Defect Versus Geometrical Reflector Discrimination in 304 Stainless Steel", Adaptronics, Inc., McLean, Virginia, October 1976.

2. Caussin, P., "U1trasonic Testing of Austenitic Stainless Steel Structures", Association Vincotte, B-1640 Rhode-Saint-Genese, Belgigue, September 1976.

3. Peterson, R. 0., Spanner, J. C., and Mech, S. J., "Development of UI traconis Method for Examining Stainless Steel Welds Interim Progress Report", HEDL-TME-75-134, Hanford Engineering Development Laboratory, Richland, Wa, November 1975.

4. McElroy, J. T., "Detailed Analysis of Fundamental UItrasonic Response Data from Stainless Steel Corrosion Crack Specimens", Final Report, EPRI Tech. Planning Study 15-26 and SURI Int. Res. Proj. 17-9183, San Antonio, TX' June 1976.

5. Holmes, E., and Beasley, D., "The Influence of Microstructure in the U1trasonic Examination of Stainless Steel Welds", J. of the Iron and Steel Inst., Vo1. 200:pp. 283-290, April 1962.

6. Herberg, G., Muller, W., and Ganglbauer, 0., "Preliminary Results for Practical Ultrasonic Testing of Austenitic Steel Welds", NDT International, pp. 239-341, October 1976.

7. Wustenburg, H. and Mundry, E., "Limiting Influences on the Reliability of U1trasonic In-Service Inspection Methods", CP8, Periodic Inspection of Fressurized Components, Instr. of Mech. Engrs., London, England, June 1974.

8. Beauchamp, K. G., Signal Processing Using Analog and Digital Techniques, Haisted Press, p. 412, 1973. 
9. Bath, Markus, "Spectral Analys is in Geophysics", Developments in Solid Earth Geophysics 7 Elsevier, p. 71, 1974.

10. Cooley, J. W., Lewis, P. A., and Weich, P. D., "Historical Notes of the Fast Fourier Transform", IEEE Transactions on Audio and Electroacoustics, Vol. Au-15, No. 2, 1967.

11. Bracewel1, R. M., The Fourier Transform and Its Applications, McGraw Hi11, p. 138, 1965.

12. Gilmore, R. S. and Czerw, G. J., "The Use of RAdiation Field Theory to Determine the Size and Shape of Unknown Reflectors by U1trasonic Spectroscopy", Materials Evaluation, Vol. 35, No. 1, pp. 37-45, January 1977.

13. Adler, L., Cook, K. V., Whaley, H. L., and Mcclung, R. H., "Flaw-Size Measurement in a Weld Sample by U1trasonic Frequency Analysis", Materials Evaluation, Vol. 35, No. 3, pp. 44-50, March 1977. 


\section{ACKNOWLEDGEMENTS}

The authors would like to thank R. G. Chafin, C. J. Fewell, 0. K. Selle, and J. S. Key for their technical assistance in conducting this program and $A$. L. Smith for interpreting the radiographic films of the tube section used in this study. We would also like to acknowledge the valuable technical discussions with Dr. W. P. Mech, Head, Math Department at Boise State Univsersity, Boise, Idaho, and the continuing support and constructive review of this program by D. F. Young. Additionally, the assistance of N. E. Dixon in supplying transducer expertise is appreciated. 